\title{
Estudio de factores causales de deserción en alumnos de ciencias veterinarias
}

\author{
Martinis-Mercado, D.; Cardozo, S.; Abraham, M.; González, C.; Sarmiento, R. \\ Facultad de Ciencias Agrarias y Veterinarias, Universidad Católica de Salta (UCASAL), \\ Campo Castañares, Salta (Argentina), tel. 54-0387-4268524. \\ E-mail:dmartinis@ucasal.edu.ar
}

\begin{abstract}
Resumen
Martinis-Mercado, D.; Cardozo, S.; Abraham, M.; González, C.; Sarmiento, R.: Estudio de factores causales de deserción en alumnos de ciencias veterinarias. Rev. vet. 27: 2, 130-133, 2016. La deserción constituye un problema importante del sistema nacional de educación formal. La Facultad de Ciencias Agrarias y Veterinarias de la UCASAL (Salta, Argentina) se propuso determinar la tasa de deserción de alumnos de veterinaria y las posibles causas que inciden en ella. Se efectuó la recolección, organización, presentación y análisis de la información, tomando como población las cohortes de los años 2009 a 2013. El análisis de los datos obtenidos estuvo basado en estadísticas descriptivas (tendencia central, dispersión y coeficiente de variación). El porcentaje de deserción resultó del 33\%. Se determinaron las causas del abandono y se formularon estrategias para su corrección. Se estima que tales medidas generarán una disminución de la deserción y por ende una mejor calidad educativa.
\end{abstract}

Palabras clave: deserción de alumnos, ciencias veterinarias, estrategias correctivas.

\begin{abstract}
Martinis-Mercado, D.; Cardozo, S.; Abraham, M.; González, C.; Sarmiento, R.: Study of desertion causal factors in veterinary sciences students. Rev. vet. 27: 2, 130-133, 2016. Desertion constitutes an important problem of the formal education national system. The Faculty of Agricultural and Veterinary Sciences of the UCASAL (Salta, Argentina) developed a research to determine the rate of veterinary science students' desertion and its possible causes. The gathering, organization, presentation and analysis of the information, was made taking as experimental population the cohorts of the years 2009 at 2013. The analysis of the obtained data was based in descriptive statistical (central tendency, dispersion and variation coefficient). The desertion percentage was of $33 \%$. Causes of the abandonment were determined and strategies for its correction were formulated. It is considered that such procedures will generate a decrease of the desertion and a better educational quality.
\end{abstract}

Key words: students desertion, veterinary studies, strategies of correction.

\section{PRÓLOGO}

Esta presentación se desprende del informe final de la investigación educacional denominada "Análisis de los factores causales de la deserción en los alumnos de la carrera de Ciencias Veterinarias (UCASAL)". La deserción es una realidad que afecta al sistema educativo argentino y que obedece a diferentes causas, dignas de conocerse a la hora de analizar dicha problemática.

Una de las manifestaciones de la crisis en la educación argentina comienza en los primeros años de la escolaridad obligatoria, cuando sólo dos de cada diez estudiantes terminan el nivel secundario ${ }^{8}$. La deserción universitaria está inserta en el mismo dilema y

Recibido: 30 marzo 2016 / Aceptado: 30 junio 2016 -por la complejidad de nuestro sistema educativo de alto nivel- resulta problemático detectar sus causas.

La deserción hace pensar sobre la calidad de los programas y modelos de enseñanza que ofrecen las instituciones superiores, el grado de preparación de los docentes, la elección de la carrera por parte del estudiante, la organización de las instituciones educativas y las diferencias entre las competencias que adquieren los alumnos en la escuela media versus las que les son requeridas en la universidad, entre otras.

El propósito de este trabajo fue plasmar una realidad que se observa consuetudinariamente, investigando y analizando las causas que llevan a un alumno a abandonar la carrera universitaria de su elección (ciencias veterinarias en este caso), estudiando la tasa de deserción de las cohortes 2009-2013, tratando de iden- 
tificar las posibles causas de abandono y proponiendo líneas de acción para reducirlas.

\section{FUNDAMENTACIÓN}

La educación superior se caracterizó, desde las últimas décadas del siglo pasado, por el notorio incremento y la heterogénea composición de su matrícula. La universidad debió abrir las puertas a sectores que hasta el momento se habían mantenido marginados de este sistema educativo. Tal característica se desencadenó, no como un evento planificado, sino como consecuencia de los cambios científico-tecnológicos, económicos, políticos y socioculturales.

Si bien entre el siglo XX y principios del siglo XXI la matrícula universitaria argentina se expandió a una tasa de crecimiento promedio del $7 \%^{2}$, un fenómeno empañó los resultados finales de estos procesos: los porcentajes de deserción fueron muy elevados y el incremento de la matrícula no fue acompañado por el consecuente aumento del número de egresados.

La deserción o tasa de abandono puede ser definida como la defección de la formación académica que ocurre por decisión personal del sujeto, independientemente de las condiciones y modalidades de las exigencias; no obedece a un retiro académico forzoso ni al generado por asuntos disciplinarios ${ }^{9}$.

Las estadísticas universitarias suelen identificar como "caso de abandono" al estudiante que ha iniciado su carrera y, antes de finalizarla, no se matricula en dos cursos consecutivos. Las causas de abandono -entre otras- pueden ser involuntarias (por incumplimiento administrativo o violación de reglamentos) o voluntarias (dejar la carrera para iniciar otra en la misma u otra institución; irse a otra universidad para completar los estudios iniciados; renunciar a la formación universitaria para escrutar itinerarios formativos fuera de la universidad, o para incorporarse al mundo laboral; o bien interrumpir la carrera con la intención de retomarla en el futuro ${ }^{1,3,4}$.

Al analizar cada caso en particular podría concluirse que algunos de ellos no son precisamente "deserciones", como en el caso de los estudiantes que abandonan la carrera elegida para iniciar otra distinta. En este caso habría ocurrido un "fracaso" académico o "frustración" vocacional. Sin embargo, para la universidad, toda situación que impulse al alumno a interrumpir sus estudios es considerada como una deserción, pues se han abandonado los objetivos académicos propuestos.

\section{DESARROLLO}

El ámbito de aplicación o magnitud sobre la cual se basó esta investigación, comprendió una población de 118 alumnos desertores (total de alumnos que abandonaron la carrera en el periodo 2009-2013). Las fuentes de información que conformaron dicha búsqueda fueron documentos institucionales (universidad) y entrevistas a los desertores (realizadas por un grupo de alumnos de la misma carrera).
El método de recolección de información se basó en encuestas (utilizando diferentes medios de recolección como ser telefónicos, informáticos [E-mails] y a través de redes sociales) que incluyeron un conjunto de preguntas "cerradas" (para poder cuantificar el análisis), las cuales se incluyen en la Tabla 1.

Tabla 1. Preguntas a efectuar y alternativas de respuestas.

1 ¿Cuál fue la orientación de la institución donde cursó el nivel secundario?

Alternativas: ciencias naturales, sociales, económicas, otras.

2 ¿Adeudaba materias pendientes al iniciar el nivel universitario?

Alternativas: si - no.

Adicionales: ¿cuántas materias? ¿cuáles?

3 ¿Cuál fue el motivo por el cual eligió la carrera de veterinaria?

Alternativas: vocación, familia, no poder trasladarse a otra provincia, otras.

4 ¿Por cuál medio averiguó aspectos de la carrera de veterinaria?

Alternativas: página web, otros medios de comunicación. amigos.

5 ¿Desarrolló actividad laboral durante el cursado de la carrera?

Alternativas: si - no.

6 ¿Fue becado por UCASAL al ingresar a la carrera de veterinaria?

Alternativas: si, - no.

7 ¿Cuáles fueron los motivos por los cuales abandonó la carrera?

Alternativas: razones económicas, laborales, no satisfizo la expectativa, el estudio le resultó complicado, no logró terminar el ciclo secundario.

8 ¿Cursa actualmente otra carrera universitaria?

Alternativas: si - no.

Adicionales: ¿cuál?, ¿dónde?

9 Durante su cursado ¿cómo hubiese calificado las áreas de la facultad?

Administración: alta - media - baja

Cuerpo docente: alta - media - baja

Personal directivo: alta - media - baja

El procesamiento de los datos se realizó a través de estadísticas descriptivas, incluyendo medidas de tendencia central (media aritmética), posición (cuartiles), dispersión (desvío estándar) y otras (variabilidad, asimetría, distribución).

\section{RESULTADOS}

Tabla 2. Tasas anuales de deserción obtenidas.

\begin{tabular}{lccc}
\hline año & ingresantes & desertores & porcentaje \\
\hline 2009 & 62 & 36 & $58 \%$ \\
2010 & 54 & 24 & $44 \%$ \\
2011 & 69 & 23 & $33 \%$ \\
2012 & 81 & 13 & $16 \%$ \\
2013 & 96 & 22 & $23 \%$ \\
total & 362 & 118 & $33 \%$ \\
\hline
\end{tabular}


En la Tabla 2 se consignan los porcentajes de deserción detectados en los cinco años de estudio, calculados en base al número de ingresantes versus desertores. Prima facie se advierte que a medida que avanzó el tiempo la tendencia al abandono de la carrera fue declinando.

La Figura 1 destaca el aumento anual del número de ingresantes a lo largo del lapso bajo estudio, así como la tendencia decreciente de la cantidad de deserciones. En efecto, durante el período 2009-2013, se registró un total de 362 alumnos ingresantes en la carrera de veterinaria, así como 118 abandonos, que representan el 33\% del total de alumnos analizados. Nótese que en el año 2009 se produjo la mayor deserción (58\%) y en 2012 la menor cantidad de abandonos (16\%).

Tabla 3. Resultado de la consulta a alumnos.

\begin{tabular}{lc}
\hline resultado & $\mathrm{n}$ \\
\hline rehusó contestar & 1 \\
cambió de carrera & 2 \\
dirección equivocada & 6 \\
ya no vive en la dirección & 21 \\
problemas de comunicación & 40 \\
encuestas contestadas & 48 \\
total general & 118 \\
\hline
\end{tabular}

La Tabla 3 expone los resultados obtenidos de las consultas ("encuestas") programadas para involucrar a 118 desertores. Se obtuvieron 48 respuestas ( $41 \%$ de los casos) contra 70 intentos frustrados por diversas causas $(59 \%)$.

El promedio (media aritmética) de alumnos desertores durante el período 2009-2013 fue de 24 por año (entre 16 y 32 según el desvío estándar. El coeficiente de variación para el período de cinco años fue del $33 \%$. El coeficiente de Bowley $(-0,81)$ indicó una asimetría hacia la izquierda de la curva de distribución de datos.

El diagrama de caja, también llamado gráfico de los 5 puntos, es la herramienta que analiza una situación general a partir del conjunto de datos de los cuartiles $1^{\circ}$ y $3^{\circ}$, la mediana y los valores máximos y mínimos. Los datos aquí obtenidos indicaron que en el cuartil $1^{\circ}$, que representa el $25 \%$ del período analizado, desertaron menos de 13 alumnos y que en el 75\% de los

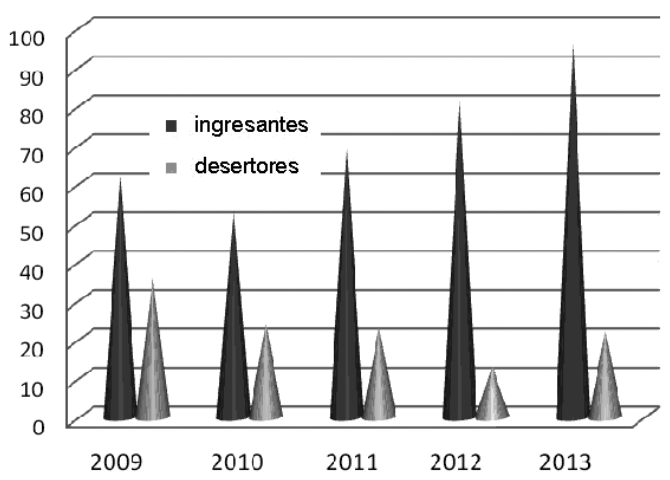

Figura 1. Alumnos ingresantes versus desertores. años analizados desertaron menos de 24 alumnos. Para los individuos encuestados se obtuvo un valor mínimo de -3 (menos tres), indicando que la situación ideal sería no haber registrado ningún alumno desertor. En el otro extremo se obtuvo un valor máximo de deserción de hasta 40 estudiantes. Por ende, tomando en cuenta los 5 años relevados, el número de estudiantes de veterinaria que abandonaron la carrera en UCASAL $(n=36)$ se ubicó dentro de los parámetros de previsibilidad obtenidos $(n=40)$.

Tabla 4. Orientación del nivel secundario.

\begin{tabular}{lc}
\hline orientación & $\%$ \\
\hline turismo & $2 \%$ \\
informática & $4 \%$ \\
carreras técnicas & $6 \%$ \\
humanísticas & $10 \%$ \\
otras & $13 \%$ \\
ciencias sociales & $15 \%$ \\
ciencias económicas & $21 \%$ \\
ciencias naturales & $29 \%$ \\
total & $100 \%$ \\
\hline
\end{tabular}

La Tabla 4 indica que las consultas a los alumnos revelaron que la mayoría de ellos (más del 70\%) había cursado el nivel secundario en instituciones con orientación desacorde a las ciencias veterinarias.

Al interrogante sobre los motivos por los cuales optó por ingresar a la carrera de veterinaria, un $77 \%$ respondió que había sido por vocación, en tanto que otros argumentaron que fue por razones familiares (4\%), por no poder trasladarse a la provincia donde se cursaba la carrera de su preferencia (4\%) u otras causas (15\%). Resulta destacable que la gran mayoría de las personas encuestadas haya ingresado a Ciencias Veterinarias por su propia preferencia, lo cual debería implicar la permanencia y continuidad de la carrera.

Los encuestados manifestaron que los medios utilizados para interiorizarse sobre la carrera fueron páginas web (22\%), otros medios de comunicación (30\%) y comentarios de sus amigos (48\%). Llama la atención que casi la mitad de los ingresantes haya decidido tan importante elección por comentarios de sus amistades. El 25\% de los consultados declaró que mientras cursó la carrera también desarrolló actividades laborales.

Indagados sobre el motivo que desencadenó el abandono de los estudios, los declarantes manifestaron que fue por problemas económicos $(50 \%)$, razones laborales $(6 \%)$, insatisfacción de sus expectativas $(25 \%)$, motivos familiares (4\%) y otros (15\%). En ningún caso el abandono ocurrió por no haber accedido al título secundario. Las causas de deserción no fueron muy diferentes de las reportadas en investigaciones similares ${ }^{6}$, 7,10 . La deserción por razones económicas (el más alto porcentaje) debería ser contemplada por la entidad académica, gestionando los medios para evitar que abandonen la carrera alumnos de buen nivel académico. 
El $60 \%$ de los estudiantes que abadonaron los estudios de veterinaria declaró que actualmente estaba cursando otra carrera universitaria, como profesorado o licenciatura en biología, abogacía, administración agropecuaria, agronomía, ciencias económicas, diseño industrial, enfermería, gestión agropecuaria, licenciatura en administración, licenciatura en nutrición, producción animal, profesorado en matemáticas, profesorado de música y varias tecnicaturas (automotriz, agroganadera, radiología, bromatología y seguridad e higiene). No fue muy halagüeño que algunos declararan que reiniciaron los estudios de veterinaria en otra universidad.

\section{COLOFÓN}

Comparada la tasa de deserción aquí obtenida (33\%) con la registrada en 2013 por el Centro de Estudios de la Educación Argentina CEA (superior al 70\%) ${ }^{11}$, surge que el porcentaje de abandono de los alumnos de la Facultad de Ciencias Agrarias y Veterinarias de la UCASAL está en niveles muy inferiores. Aquélla tasa del 70\% implica que solamente egresan 27 de cada 100 estudiantes que inician una carrera universitaria ${ }^{11}$.

Otros investigadores afirman que en las universidades públicas de Argentina las tasas de deserción son del $43 \%$ en mujeres y del $46 \%$ en hombres. En las universidades privadas el guarismo sería menor (alrededor del 39\% ${ }^{5}$. El porcentaje obtenido en este trabajo (33\%) hace pensar que la carrera de ciencias veterinarias de la Facultad de Salta consta con un índice de deserción inferior al de otras universidades privadas.

No obstante ello, en UCASAL se han implementado acciones capaces de reducir aún más la tasa en cuestión, como la implementación de un sistema de ayuda a los alumnos por parte de docentes tutores y pares tutores (alumnos). Tales estrategias se completan con becas de ayuda económica para los estudiantes no pudientes que registren buen rendimiento académico. Se estima que tales iniciativas tiendan a reducir o eliminar el flagelo de la deserción estudiantil.
Agradecimientos. Por la colaboración prestada, a los alumnos de la Facultad de Ciencias Agrarias y Veterinarias de la Universidad Católica de Salta, Marisel Barroso, Sergio Suares y Agustín Avellaneda.

\section{REFERENCIAS}

1. Álvarez-Pérez P, Cabrera L, González M, Benítez J. 2006. Causas de abandono y prolongación de los estudios universitarios. Paradigma (Maracay) 27: 1.

2. García-Fanelli A. 2005. Acceso, abandono y graduación en la educación superior argentina. Sistema de información de tendencias educativas en América Latina, UNESCO, IIPE, OEI. http://cippec.org/files/documents/ Otras\%20Publicaciones/de mocratizarelacceso_EDU.pdf

3. González M, Álvarez P, Bethencourt J, Cabrera, L. 2005. Abandono y prolongación de los estudios universitarios: un obstáculo en la mejora de la calidad de la enseñanza. Rev Electr Invest \& Eval Educ 12: 2.

4. González M, Cabrera L, Tomas J, Alvarez P. 2006. El problema del abandono de los estudios universitarios. Rev Relieve 12: 171-203.

5. Landi J. 2001. Los desertores no son tantos. Investigación sobre graduación en la universidad. www.pagina12.com. ar/2001/01-06/01-06-26/univer01.htm

6. López N, Tedesco JC. 2002. Las condiciones de educabilidad de los niños y adolescentes de América Latina. Publ. Instit Intern Planeam Educ (UNESCO). http://www.udelas.ac.pa/biblioteca/librospdf/educabilidad.PDF

7. Messing C. 2007. Desmotivación, insatisfacción y abandono de proyectos en los jóvenes, Ed. Noveduc, Buenos Aires, 328 p.

8. Mollis M. 2001. La universidad argentina en tránsito, Ed. Fondo de Cultura Económica (FCE), Buenos Aires, 144 p.

9. Páramo GJ, Correa CA. 1997. Deserción estudiantil universitaria. Conceptualización. Publicaciones.eafit.edu.co/ index.php/revista...eafit/../967\%3A\%3Apdf

10. San Martin R. 2001. Deserción Universitaria. Artículo del diario La Nación, Buenos Aires, 1-2-2001.

11. Sánchez F. 2013. Universidades: se reciben solo 27 alumnos de 100 que ingresan. Artículo del diario La Nación, Buenos Aires, 4 de junio de 2013. 